\title{
ON THE GENUS OF SYMMETRIC GROUPS
}

BY

\author{
VIERA KRŇANOVÁ PROULX
}

\begin{abstract}
A new method for determining genus of a group is described. It involves first getting a bound on the sizes of the generating set for which the corresponding Cayley graph could have smaller genus. The allowable generating sets are then examined by methods of computing average face sizes and by voltage graph techniques to find the best embeddings.

This method is used to show that genus of the symmetric group $S_{5}$ is equal to four.

The voltage graph method is used to exhibit two new embeddings for symmetric groups on even number of elements. These embeddings give us a better upper bound than that previously given by $A$. T. White.
\end{abstract}

A Cayley graph corresponding to a generating set $S$ of a group $G$ consists of a vertex set labeled by the elements of the group $G$ and a set of directed labeled edges, $=\{(u g v) \mid u, v \in G, g \in S, u g=v\}$. The genus of a group $G$ is defined to be minimal genus among all its Cayley graphs.

The purpose of this paper is to present a new method for determining the genus of a group. It works like this. First, we use the method of voltage graphs or any other method to get a reasonably good bound on the genus of this group. We then employ Theorem 1 to get a bound on the size and conditions on the structure of a generating set for which the corresponding Cayley graph could have smaller genus. We then have to look at all allowable generating sets and use the method of computing the average face sizes either to find a better embedding or to show that none exists.

This method is still very lengthy and can only be used for a few specific groups for which determining the genus seems to be particularly important. We use it here to compute the genus of $S_{5}$, the symmetric group on five elements, which is the smallest nonplanar symmetric group. (Even the alternating group $A_{5}$ is planar (Maschke [4]).) However, these methods were the main tool used in classifying all toroidal groups (Proulx [5], [6]) and have already been used in obtaining further important results concerning the genera of groups (Tucker [7]).

The first part of this paper introduces a theorem which describes the relationship between the size of a generating set $S$ of a group $G$ and the genus of the corresponding Cayley graph. This theorem is used in $\$ 2$ where we prove that the genus of the symmetric group $S_{5}$ is four, which equals the upper bound on the

Received by the editors November 5, 1979 and, in revised form, July 29, 1980.

1980 Mathematics Subject Classification. Primary 05C10; Secondary 05C25, 57M15, 20 F32.

Key words and phrases. Cayley graph, genus of a group, symmetric group, voltage graphs, Euler characteristic. 
genus of $S_{5}$ given by White [9]. The proof mainly uses the method of computing the average face sizes, and occasionally also the method of voltage graphs for finding graph embeddings (Gross [2]). The voltage graph method is used again in $\$ 3$ to exhibit two new embeddings for symmetric groups on $n$ elements $S_{n}$ where $n$ is even. These embeddings give us a better upper bound on the genus of $S_{n}$ than that previously known (White [9]).

0. Preliminaries. 1. The generators of a group $G$ will be denoted either by the letters $a, b, c, d$ or by the letters $g_{1}, g_{2}, g_{3}, \ldots$ The words in these generators will be denoted by letters $u, v$, and $w$.

2. We will use numerous times the Euler polyhedral equation $V-E+F=\chi$ for embeddings of graphs on surfaces. Here $V$ is the number of vertices, $E$ is the number of edges, and $F$ is the number of faces in the embedding of this graph on a surface of Euler characteristic $\chi$. For orientable surfaces of genus $g$ we have $\chi=2-2 g$. That implies that if $\chi$ is odd, the embedding must be nonorientable.

3. It should be noted that we only need to look at the minimal generating sets (i.e. with no redundant generators). Deleting a redundant generator corresponds to deleting edges in the Cayley graph, which cannot increase the genus.

4. We say that a relator $w$ is a reduced relator in the group $G$ if $w$ contains no nontrivial subword that is a relator in $G$. Clearly, the product of labels along a boundary of a face in an embedding of a Cayley graph has to be a reduced relator in $G$. (When traversing the edge ( $u g v$ ) from $v$ to $u$ we read the label as $g^{-1}$.)

5. The words $w_{1}$ and $w_{2}$ are said to be cyclically equivalent $w_{1} \sim w_{2}$ if and only if there exist words $u_{1}, u_{2}$ such that $w_{1}=u_{1} u_{2}$ and either $w_{2}=u_{2} u_{1}$ or $w_{2}=\left(u_{2} u_{1}\right)^{-1}$. It can be shown that this is an equivalence relationship. Also if $w_{1}$ is a relator in $G$ and $w_{1} \sim w_{2}$ then $w_{2}$ is also a relator in $G$.

1. The main theorem. A Cayley graph of a group $G$ of order $|G|=V$ corresponding to a generating set $S=\left\langle g_{1}, g_{2}, g_{3}, \ldots, g_{r}\right\rangle$ is a regular graph of degree $2 r$ with $V$ vertices. One would expect that the more edges a graph with a given number of vertices has, the harder it will be to find an embedding of this graph on a surface of small genus. So in order to determine the genus of a group one would only need to examine the Cayley graphs corresponding to "small" generating sets. This idea is substantiated by Theorem 1 .

THeOREM 1. Let $G$ be a group with a generating set $S=\left\{g_{1}, g_{2}, \ldots, g_{r}\right\}$, such that $k$ generators are of order two, $m$ generators are of order three and $n$ generators are of order greater than three. (Of course $k+m+n=r$.) Then a necessary condition for the Cayley graph corresponding to the generating set $S$ to have an embedding in a surface of Euler characteristic $\chi$ is that

$$
k+5 m / 3+2 n \leqslant 4(|G|-\chi) /|G| \text {. }
$$

Proof. The Cayley graph corresponding to a minimal generating set $S=$ $\left\{g_{1}, g_{2}, \ldots, g_{r}\right\}$ of the group $G$ has $V=|G|$ vertices and

$$
E=r V=(k+m+n)|G|
$$


edges. To get the desired characteristic $\chi$ the embedding will have to have $F=\chi-V+E=2-2 g-V+E$ faces. So the smaller the genus the more faces we need in the embedding while the number of edges remains constant. Therefore the average face size $A=2 E / F$ will have to be small in an embedding on a surface of small genus.

The smallest possible faces in a Cayley graph are digons with labels $g_{1}^{2}, g_{2}^{2}, \ldots, g_{k}^{2}$. There can be at most $k|G| / 2$ of them using up $k|G|$ edges. We can also have at most $m|G| / 3$ triangles with labels $g_{k+1}^{3}, g_{k+2}^{3}, \ldots, g_{k+m}^{3}$ using up $m|G|$ edges. If we use all of these faces in an embedding on a surface of Euler characteristic $\chi$ we will have $2 E^{\prime}=2 E-k|G|-m|G|=|G|(k+m+2 n)$ edges left to build

$$
F^{\prime}=F-k|G| / 2-m|G| / 3=\chi-|G|+|G|(k+m+n-k / 2-m / 3)
$$

faces. But the average face size for the remaining faces must be greater than or equal to four which implies

$$
2 E^{\prime} / F^{\prime}=|G|(k+m+2 n) /(\chi-|G|+|G|(k / 2+2 m / 3+n))>4 \text {. }
$$

From that follows

$$
(k+5 m / 3+2 n) \leqslant 4(|G|-\chi) /|G| \text {. Q.E.D. }
$$

2. Genus of the symmetric group $S_{5}$. This section consists entirely of the proof of Theorem 2.

THEOREM 2. The genus of the symmetric group $S_{5}$ is four.

Proof. It has been mentioned that the genus of $S_{5}$ is at most four. G. A. Jones (personal communication) has shown that all toroidal groups are solvable, and so $g\left(S_{5}\right) \geqslant 2$. Alternatively, methods such as those of Gross and Lomonaco [3] can be used to show that $S_{5}$ is not toroidal. From Theorem 1 and the fact that $g\left(S_{5}\right)<4$ it follows that $(k+5 m / 3+2 n) \leqslant 4$. This implies that a generating set $S$ of the group $S_{5}$ for which the corresponding Cayley graph has the minimal genus must consist of either

(i) two generators or

(ii) three generators, at least two of which are of order two or

(iii) four generators, all of which have order two.

The rest of the proof is mostly a very tedious examination of all possible generating sets with two, three or four generators. It uses heavily the fact that each face boundary is labeled by a reduced relator and therefore, for a given generating set $S$, there cannot be a face of size $t$ unless there exists a reduced relator of length $t$.

Case (iii). Four generators. This case is not too long and can be done here completely.

A Cayley graph corresponding to the generating set $\left\{a, b, c, d \mid a^{2}=b^{2}=c^{2}=\right.$ $\left.d^{2}=e\right\}$ consists of 120 vertices and 480 edges, each appearing on a boundary of a face exactly twice. To get the best embedding one needs to maximize the number of faces, and therefore to minimize their sizes. The smallest faces are digons 
$a^{2}, b^{2}, c^{2}, d^{2}$. If all 240 of them appear in an embedding they will use up 480 edges. That will leave 480 edges not yet used as face boundaries. These will have to be used to build 118,116 , or 114 faces to get embeddings of genus two, three, or four respectively, with the average face sizes $4.07,4.13$, or 4.21 . There are no relators of length three or five. So to get 114 faces, at least 102 must be quadrangles (the rest would be 12 hexagons, or 6 octagons and 6 quadrangles, or some other possibilities). To get 116 faces, at least 108 must be quadrangles (and 8 hexagons or so); and similarly of the 118 faces at least 114 must be quadrangles (and 4 hexagons). If each vertex was adjacent to only three quadrangles, we could get only $(120 \times 3) / 4$ $=90$ quadrangles. So there must be a vertex $v$ adjacent to four quadrangles. Suppose the four generators were $a, b, c, d$. Then the neighborhood of the vertex $v$ would be as shown in Figure 1. That means the following must hold: $(a b)^{2}=(b c)^{2}$ $=(c d)^{2}=(a d)^{2}=e$. But there is no generating set of $S_{5}$ satisfying this condition. (Choosing $a=(12)$ and $b=(34)$ forces $c=(25)$, but then there is no suitable $d$. Choosing double transpositions just makes things worse.) That means that a Cayley graph corresponding to a generating set of $S_{5}$ of size four with all generators of order two must have a genus greater than four.

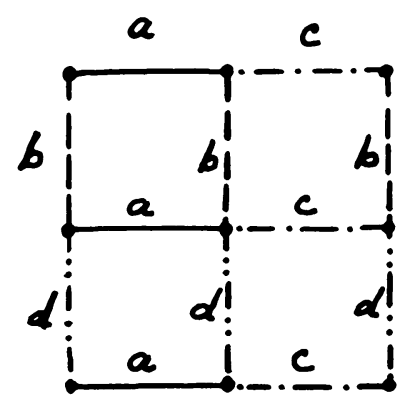

Figure 1

Case (i). Two generators. Two generators of order two cannot generate $S_{5}$. They generate instead a dihedral group $D_{k}$ where $k$, the order of the product, must be of order greater than two. Table 1 is a list of all possible combinations of an element of order two and another element of $S_{5}$ of order greater than two, such that at least one of them is odd and together they involve all letters in nondisjoint cycles.

\section{TABLE 1}

$\begin{array}{llll}A & a=(12) & b=(2345) & a b=(13452) \\ B & a=(12) & b=(12345) & a b=(1345)(2) \\ C & a=(12) & b=(13)(245) & a b=(14523) \\ D & a=(12)(34) & b=(2345) & a b=(1352)(4) \\ E & a=(12)(34) & b=(2354) & a b=(132)(45) \\ F & a=(12)(34) & b=(123)(45) & a b=(1354)(2) \\ G & a=(12)(34) & b=(135)(24) & a b=(145)(23)\end{array}$


The Cayley graphs corresponding to these generating sets all have $V=120$ vertices, $E=240$ edges and we need $F=114,116$, or 118 faces to get an embedding of genus 4.3 , or 2 respectively. Of course, each edge appears on a boundary of two faces. To maximize the number of faces one must minimize the face sizes. The smallest faces in all these cases are digons with boundary $a^{2}$. There are 60 of them and they use up 120 edges. That leaves us with 360 edges to build 54,56 , or 58 faces.

It turns out that case $D$ leads to a toroidal embedding of the derived voltage graph one gets by lifting a bouquet of two circles in Figure 2 using a voltage group $S_{5}$. Therefore, this cannot be a generating set of $S_{5}$.

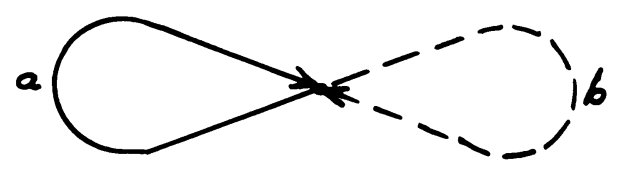

FIGURE 2

In cases $A$ and $E$ we first use all faces of type $(a b)^{5}$ and $(a b)^{6}$, respectively, and see that the average face size for the remaining faces would have to be at most 8.64 (resp. 10.0). In order to find all relators of length ten or less it is only necessary to look at representatives of all classes of cyclically equivalent words of lengths less than or equal to ten. It can be shown that none of these words is a relator except $(a b)^{5}$ in case $A$ and $\left(a b^{2}\right)^{3}$ in case $E$. This is a tedious process and will not be printed here. The author will supply the details upon request to anyone interested. In case $A$ lifting of the voltage graph in Figure 2 results in an embedding of the Cayley graph of $S_{5}$ on a surface of genus four. The best that can be done in case $E$ is an embedding of genus 11 by lifting the voltage graph in Figure 3. All faces have size nine with boundary $\left(a b^{2}\right)^{3}$.

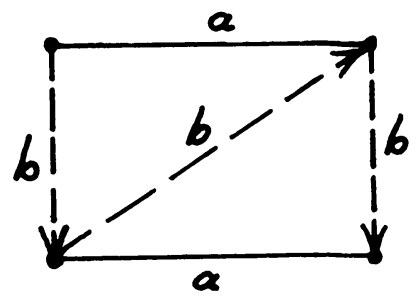

FIGURE 3

In case $B$ there are no quadrilateral faces possible. The voltage graph of Figure 1 will again lift to a genus four embedding which is the best possible. There are no relators of length five except $b^{5}$. Using them leaves 240 edges to build 34,32 , or 30 faces with average face sizes $7.06,7.50$, and 8.00 . No other word of length eight or less is a relator (except $(a b)^{4}$ considered earlier).

In the remaining cases (i.e. $C, F$, and $G$ ) the generator $b$ is of order six. Considerations similar to those above show that there are no other relators of 
length six or less. If all 20 faces $b^{6}$ are used, the average face size for the remaining faces will have to be $6.31,6.66$, or 7.06 respectively for a genus four, three, or two embedding. But there are no relators of length seven either and thus no embeddings of Cayley graph of $S^{5}$ of genus less than four with these generating sets.

Next consider the case when one of the generators is of order three. There are four such generating sets as listed in Table 2. If all 40 faces $b^{3}$ are used in an embedding of genus four, three, or two the average face size of the remaining faces has to be $4.09,4.18$, or 4.29 respectively. But the only relators of length four are $b^{4}$ in cases $A$ and $B$. However that requires that there exist an additional relator of length five or less which is not the case. Thus, none of these four generating sets results in an embedding of genus four or less.

\section{TABLE 2}

$\begin{array}{llll}A: & a=(123) & b=(1245) & a b=(145)(23) \\ B: & a=(123) & b=(1425) & a b=(15)(243) \\ C: & a=(123) & b=(12)(345) & a b=(1)(2453) \\ D: & a=(123) & b=(14)(235) & a b=(134)(25)\end{array}$

At last let us consider the generating sets of size two with both generators of order four or more. The smallest faces possible are quadrilaterals and there must be at least 110,100, or 90 of them to get embeddings of genus two, three, or four respectively. For genus four this is achieved with the generating set $S=\{a, b \mid a=$ (1234), $b=$ (15432), $a b=$ (1) (2) (3) (45) $\}$. The embedding uses 30 faces $a^{4}, 60$ faces $(a b)^{2}$, and 24 faces $b^{5}$. To get an embedding of genus two or three, even more quadrilateral faces are needed. But they could only have boundary $b^{4}$. Unfortunately, using all 30 quadrilateral faces would result in a toroidal embedding which contradicts the fact that $S^{5}$ has genus at least two. This concludes case (i) of the proof of Theorem 2.

Case (ii). Three generators. Consider now the generating sets $S$ of $S^{5}$ consisting of three generators, two of which must have order two, i.e., $S=\left\{a, b, c \mid b^{2}=c^{2}=\right.$ $e, a^{r}=e$ where $r=6,5,4,3$, or 2$\}$.

The smallest faces that are used here are digons $b^{2}$ and $c^{2}$, sixty of each kind. Actually one can just visualize replacing each of the digons $b^{2}$ and $c^{2}$ with a single nonoriented edge labeled $b$ or $c$. Now there are 480 edges left to build 118, 116, or 114 faces to get an embedding of genus 4, 3, or 2 respectively.

If $a$ is of order greater than three, the smallest faces possible are quadrilaterals. There are at least 110,100 , or 90 of them needed to get an embedding of genus four, three, or two, respectively. But that means that there are at least three quadrilaterals adjacent to each vertex. A neighborhood of a vertex can be embedded in essentially two ways shown in Figure 4 below. That means that one of the following conditions must be satisfied by a generating set $S=\{a, b, c\}$ whose Cayley graph is of genus four or less.

Condition $C 1$. At least three words from the set

$$
W=\left\{a^{4},(a b)^{2}, a b a b^{-1},(b c)^{2},(a c)^{2}, a c a^{-1} c\right\}
$$

are relators. 
Condition $C 2$. At least two words from the set $U=\left\{(a c)^{2}, a c a^{-1} c,(a b)^{2}, a b a^{-1} b\right\}$ are relators.
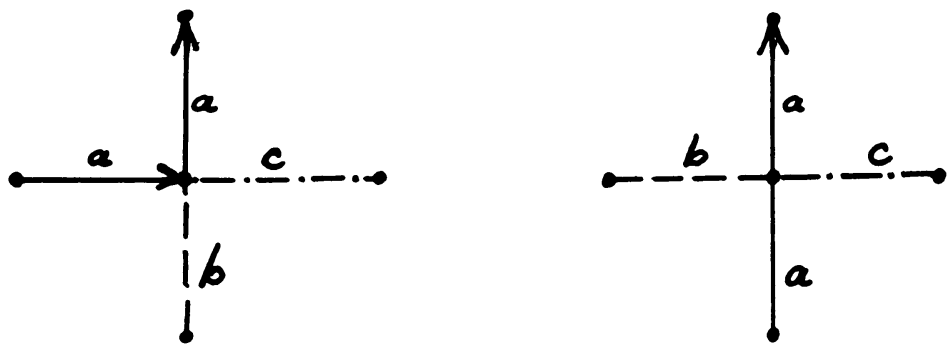

FIGURE 4

One can then consider six separate cases: when the generator $a$ is of order six, five, four or three, and two cases when $a$ is of order two. In one of them two of the generators are double transpositions, in the other there is only one double transposition. Only one of the Cayley graphs corresponding to these generating sets has genus four, none have smaller genus. The Cayley graph corresponding to the generating set $a=(12), b=(12)(34), c=(13)(25)$ has a nonorientable embedding of genus four with faces $a^{2}, b^{2}, c^{2},(a b)^{2},(a c)^{4},(b c)^{5}$.

This concludes the proof that the genus of the symmetric group on five elements $S_{5}$ is equal to four.

3. Symmetric group $S_{n}$ with $n$ even. We conclude by presenting two new embeddings for the symmetric group on an even number of elements ( $S_{n}$ where $n$ is even and less than 168). The best embeddings known so far were given by White [9]. They correspond to the generating set $S=\{a, b \mid a=(1,2), b=(1,2, \ldots, n)\}$ and use faces $a^{2}, b^{n}$ and $(a b)^{n-1}$. The genus of these embeddings is

$$
g=1+\frac{(n-2) !}{4}\left(n^{2}-5 n+2\right)
$$

A Cayley graph of $S_{n}$ for $n$ even corresponding to the generating set $S=\{a, b \mid a=$ $(1,2), b=(2,3, \ldots, n)\}$ has an orientable embedding in a surface of genus

$$
g=1+\frac{n !}{6} \cdot \frac{(n-4)}{(n-1)}
$$

For $n>12$ this is a better bound than the one given by (3.1). This embedding is obtained by lifting the voltage graph in Figure 5 into two isomorphic components each of which will be an embedding of the desired voltage graph. Observe that $\left(a b a^{-1} b^{-1}\right)^{3}=e\left[1\right.$, p. 63]. Thus in each component there will be $n ! / 2$ faces $a^{2}$, $n ! /(n-1)$ faces $b^{n-1}$, and $n ! / 6$ faces $\left(a b a^{-1} b^{-1}\right)^{3}$. The reason that this voltage graph lifts into two disjoint copies of the Cayley graph of $S_{n}$ is that it is a Schreier coset graph for a quotient group $S_{n} / A_{n}$. 


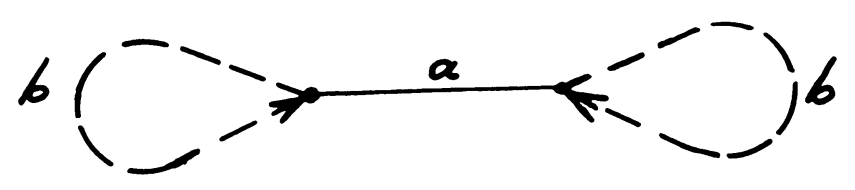

Figure 5

An even better bound on the genus of $S_{n}$ for $n$ even can be obtained by lifting the voltage graph in Figure 6 when the generating set is $S=\{a, b \mid a=(1,2)$, $b=(1,2, \ldots, n)\}$. The derived graph will again contain two isomorphic copies of the desired Cayley graph. There will be $n ! / 2$ faces $a^{2}$ and $n ! / 4$ faces $\left(a b^{2} a b^{-2}\right)^{2}$ in each component. (See [1, p. 63].) This embedding has genus

$$
g=1+\frac{n !}{8} \text {. }
$$

This is better than the bound (3.1) for $n \geqslant 10$ and better than (3.2) for all positive $n$.

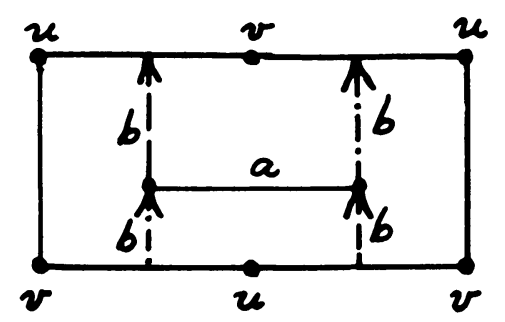

Figure 6

ADDED IN PROOF (August 26, 1980). Tucker [8] just recently has determined the genus of $S_{n}$ for $n \geqslant 168$ to be

$$
\gamma\left(S_{n}\right)=\frac{n !}{168}+1
$$

However, for $n<168$ the exact genus is still unknown.

\section{REFERENCES}

1. H. S. M. Coxeter and W. O. J. Moser, Generators and relations for discrete groups, 3rd ed., Ergebnisse der Math. und ihrer Grenzgebiete, Bd. 14, Springer-Verlag, Berlin, 1972.

2. J. L. Gross, Voltage graphs, Discrete Math. 15 (1974), 239-246.

3. J. L. Gross and S. J. Lomonaco, Jr., A determination of the toroidal K-metacyclic groups, J. Graph Theory 4 (1980).

4. H. Maschke, The representation of finite groups, Amer. J. Math. 18 (1896), 156-194.

5. V. K. Proulx, Classification of the toroidal groups, Ph.D. Thesis, Columbia Univ., 1977.

6. _ Classification of the toroidal groups, J. Graph Theory 2 (1978), 269-273.

7. T. W. Tucker, The number of groups of given genus, Trans. Amer. Math. Soc. 258 (1980), 167-179.

8. ___ Some results on a genus of a group, J. Graph Theory (submitted).

9. A. T. White, On the genus of a group, Trans. Amer. Math. Soc. 173 (1972), 203-214.

Department of Mathematics, Northeastern University, Boston, Massachusetts 02115 